de los recursos turisticos de la provincia de Tarma

\title{
LA PUBLICIDAD NO CONVENCIONAL (BTL) COMO MEDIO ALTERNATIVO PARA LA DIFUSIÓN DE LOS RECURSOS TURISTICOS DE LA PROVINCIA DE TARMA
}

\section{NON CONVENTIONAL ADVERTISING (BTL) AS AN ALTERNATIVE MEAN FOR THE DISSEMINATION OF THE PROVINCE OF TARMA TOURISM RESOURCES}

\section{RESUMEN}

\author{
Antonio Palomino Crispín ${ }^{20}$, Ketty Egoavil Guillermo ${ }^{21}$
}

Instituto de Investigación de Ciencias Aplicadas - Tarma

El trabajo de investigación titulado La publicidad no convencional (btl) como medio alternativo para la difusión de los recursos turísticos de la provincia de Tarma", parte de la necesidad de reconocer los medios utilizados por los operadores turísticos, con la siguiente interrogante ¿es la estrategia de publicidad no convencional un medio alternativo de importancia para la promoción de los recursos turísticos de la provincia de Tarma?. Su objetivo general es demostrar que la estrategia de publicidad no convencional es un medio alternativo de importancia para la promoción de los recursos turísticos de la provincia de Tarma.. Por lo cual se plantea la siguiente hipótesis: La estrategia de publicidad no convencional se convierte en un medio alternativo de importancia para la promoción de los recursos turísticos de la provincia de Tarma. La investigación es de tipo descriptivo, nivel no experimental, se utilizará el método científico y como métodos específicos: el deductivo e inductivo y las técnicas de observación entrevista, cuestionario y fichaje. El impacto esperado es la optimización del empleo de los medios que utiliza la publicidad no convencional en la difusión de los recursos turísticos por parte de los operadores de ese sector importante de la economía de la provincia de Tarma.

Palabras clave: publicidad, no convencional, turismo

\begin{abstract}
The paper titled unconventional advertising (BTL) as an alternative means for the dissemination of tourism resources of the province of Tarma, "part of the need to recognize the means used by tour operators, with the following question Is unconventional advertising strategy alternative means of importance for the promotion of tourism resources of the province of Tarma?. Whose overall objective is to demonstrate that the unconventional advertising strategy is an alternative means of importance for the promotion of tourism resources of the province of Tarma. Therefore the following hypothesis is posed: The unconventional advertising strategy becomes an important alternative mean for the promotion of tourism resources of the province of Tarma. The research is descriptive, not experimental level will be used as the scientific method and specific methods: deductive and inductive observation and interview techniques, questionnaire and signing. The expected impact is to optimize the use of media that uses unconventional advertising dissemination of tourism resources by operators in that sector of the economy of the province of Tarma.
\end{abstract}

Key words: publicity, not conventional, tourism.

20 Email: king_eleodoro@hotmail.com

21 Email: ketita15@hotmail.com 


\section{INTRODUCCIÒN}

Cuando se habla de "below the line" se están agrupando las acciones de comunicación que se conocen también como publicidad no convencional, es decir, las acciones que no involucren a los medios tradicionales como la $\mathrm{TV}$, radio, medios impresos, etc.

Dentro del below the line (o publicidad no convencional) se ubican el marketing directo, el marketing promocional (las promociones de ventas), el marketing relacional: las relaciones públicas, patrocinios, merchandising, etc., donde no se emplean medios masivos.

EI BTL 0 below the line (debajo de la línea), es una estrategia del marketing, que ya tiene algunas décadas, sin embargo, parece que hoy en día es algo innovador. Puede o no ser complemento del ATL 0 above The line sobre la linea. Algo muy curioso es que el BTL está en vigor mucho antes que apareciera el ATL. Solo que hoy en dia a tenido tanta relevancia por su efectividad, a la hora de aplicarlo a un producto y/o servicio.

Por otro lado en función a la difusión de los recursos turísticos, es de un tratamiento especial realizar el enfoque correspondiente, puesto que está referida enteramente a la utilización de medios de carácter no convencional o los meramente llamados clásicos, en donde se tendrá especial atención en los medios de contacto personal y menos masivo en su comunicación.

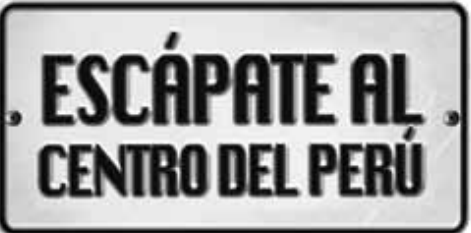

Figura 1.- Viajemos al centro del Perú

El objetivo tiene el propósito de demostrar que la publicidad no convencional es un medio alternativo de importancia para la promoción de los recursos turísticos de la provincia de Tarma.

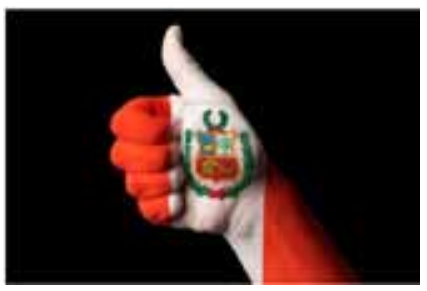

Imagen 2.- Marca Perú

\section{Y los objetivos específicos}

- Realizar un análisis y diagnóstico de la publicidad en forma general empleado para la promoción de los recursos turísticos de la provincia de Tarma.

- Reconocer los mecanismos y medios de publicidad no tradicionales, utilizados como medio alternativo para la promoción de los recursos turísticos de la provincia de Tarma

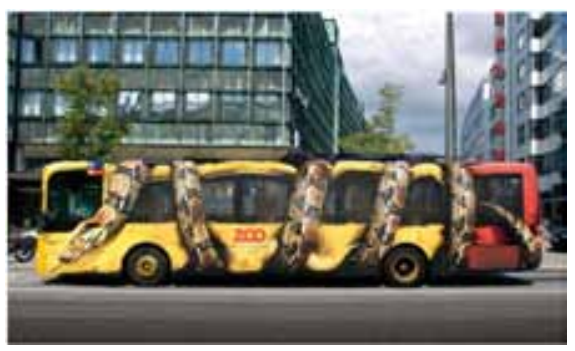

Figura 3.- visitemos el zoológico

\section{MATERIALES Y MÉTODOS}

Es de carácter descriptivo porque tiene como propósito identificar las formas y medios empleados dentro de la publicidad no convencional como medio alternativo para la difusión de los recursos turísticos de la provincia de Tarma, para ello se recurrió a los agentes y operadores turísticos quienes contacto directo con la demanda de turistas a la provincia de Tarma.

Para dicho propósito se estructuró el cuestionario pertinente que permita reconocer el conocimiento de la publicidad no convencional y su empleo así como la programación de las entrevistas con sus 
conductores y/o representantes.

Para este propósito, se determinó como muestra/ universo a la totalidad de operadores turísticos (específicamente agencias de viajes) que suman en número de 20 establecimientos entre permanentes y eventuales, considerando las temporadas altas de funcionamiento de dichos establecimientos.

Teniendo en cuenta la finalidad y el tipo de investigación, el estudio es de nivel no experimental.

\section{Método: deductivo y inductivo}

\section{Técnicas:}

Para la investigación se utilizarán las siguientes técnicas:

- Observación

- Entrevista (Operadores turísticos,)

- Cuestionario

- Fichaje

\section{RESULTADOS}

\section{EN RELACION A LA PUBLICIDAD}

Siendo ésta, una investigación relacionada a la publicidad no convencional como medio alternativo para la difusión de los recursos turísticos de la provincia de Tarma, esta primera parte muestra los resultados a nivel del conocimiento del entorno de la oferta y demanda, haciendo hincapié en la publicidad.

Tabla $N^{\circ}$ 1. Cuán importante considera a la publicidad en la difusión de recursos turísticos.

\begin{tabular}{lll}
\hline \multicolumn{1}{c}{ Condición } & & $\%$ \\
\hline Demasiado importante & 10 & 50 \\
Bastante importante & 8 & 40 \\
Poco importante & 2 & 10 \\
Nada importante & & \\
\hline
\end{tabular}

En tal sentido en el cuadro $\mathrm{N}^{0} 1$, se puede apreciar que, en relación al nivel de importancia de la publicidad en difusión de recursos turísticos, existe una inclinación en un $50 \%$ en considerar demasiado importante y $40 \%$ en considerar bastante importante, en relación a un $10 \%$ que considera poco importante.

Tabla N² 2. Conoce a su público objetivo.

\begin{tabular}{lll}
\hline \multicolumn{1}{c}{ Condición } & & $\%$ \\
\hline Conoce bastante & 2 & 10 \\
Conoce parcialmente & 9 & 45 \\
Conoce mínimamente & 8 & 40 \\
desconoce & 1 & 20 \\
\hline
\end{tabular}

En tal sentido en cuadro $\mathrm{N}^{\circ} 2$, se puede apreciar que, en relación al conocimiento de su público objetivo para la difusión de recursos turísticos, existe un conocimiento en $45 \%$ en conocimiento parcial y $40 \%$ en conocimiento mínimamente ante un $10 \%$ que conoce bastante.

Tabla $\mathrm{N}^{\circ} 3$. Tiene conocimiento o capacitación en publicidad.

\begin{tabular}{lcc}
\hline \multicolumn{1}{c}{ Condición } & & $\%$ \\
\hline Abundante & 1 & 5 \\
Bastante & 8 & 40 \\
Poco & 8 & 40 \\
Nada & 3 & 15 \\
\hline
\end{tabular}

El cuadro $N^{0} 3$, se puede apreciar que, en relación al nivel de conocimiento y capacitación de publicidad en difusión de recursos turísticos, existe un $40 \%$ que conoce bastante e igualmente $40 \%$ que conoce poco en relación a un $115 \%$ que no conoce nada.

Tabla $N^{\circ} 4$. Conoce los recursos turísticos de la provincia de Tarma.

\begin{tabular}{lcc}
\hline \multicolumn{1}{c}{ Condición } & $\%$ \\
\hline Abundante & 18 & 90 \\
Bastante & 2 & 10 \\
Poco & & \\
Nada & & \\
\hline
\end{tabular}

El cuadro $N^{\circ} 4$, se puede apreciar que, en relación al nivel de conocimiento de recursos turísticos, existe una inclinación en un $90 \%$ en conocer abundantemente y $10 \%$ en conocer bastante. 
Tabla N 5. Cuán importante considera la asignación permanente de un presupuesto específico para la publicidad en la difusión de recursos turísticos.

\begin{tabular}{lcc}
\hline \multicolumn{1}{c}{ Condición } & $\%$ \\
\hline Demasiado importante & 2 & 10 \\
Bastante importante & 12 & 60 \\
Poco importante & 5 & 25 \\
Nada importante & 1 & 5 \\
\hline
\end{tabular}

El cuadro $\mathrm{N}^{0} 5$, se puede apreciar que, en relación al nivel de importancia de la asignación de un presupuesto especifico para la publicidad en difusión de recursos turísticos, existe una inclinación en un $60 \%$ en considerar bastante importante y $25 \%$ en considerar poco importante, en relación a un $5 \%$ que considera nada importante.

\section{EN RELACION AL EMPLEO DE LOS MEDIO UTILIZADOS}

Tabla $N^{\circ} 6$. Cuál es el nivel de empleo del marketing directo en la publicidad y la difusión de recursos turísticos.

\begin{tabular}{lcc}
\hline \multicolumn{1}{c}{ Condición } & & $\%$ \\
\hline Emplea abundantemente & 1 & 5 \\
Emplea frecuentemente & 4 & 20 \\
Emplea parcialmente & 4 & 20 \\
Emplea mínimamente & 11 & 55 \\
\hline
\end{tabular}

El cuadro $N^{\circ} 6$, se puede apreciar que, en relación al nivel de empleo del marketing directo de la publicidad en la difusión de recursos turísticos, existe una inclinación en un $20 \%$ en empleo frecuente y $20 \%$ en empleo parcialmente en relación a un $55 \%$ que emplea mínimamente.

Tabla $N^{\circ} 7$. Nivel de empleo de la promoción de ventas en la publicidad y la difusión de recursos turísticos.

\begin{tabular}{lcc}
\hline \multicolumn{1}{c}{ Condición } & & $\%$ \\
\hline Emplea abundantemente & 10 & 50 \\
Emplea frecuentemente & 7 & 35 \\
Emplea parcialmente & 2 & 10 \\
Emplea mínimamente & 1 & 5 \\
\hline
\end{tabular}

El cuadro $N^{0} 7$, se puede apreciar que, en relación al nivel de empleo de la promoción de ventas de la publicidad en la difusión de recursos turísticos, existe una inclinación en un $50 \%$ en empleo abundante y $35 \%$ en empleo frecuente, en relación a un $5 \%$ que emplea minimamente.

Tabla $\mathrm{N}^{\circ}$ 8. Nivel de empleo del merchandising en la publicidad y la difusión de recursos turísticos.

\begin{tabular}{lll}
\hline \multicolumn{1}{c}{ Condición } & & $\%$ \\
\hline Emplea abundantemente & 8 & 40 \\
Emplea frecuentemente & 6 & 30 \\
Emplea parcialmente & 4 & 20 \\
Emplea mínimamente & 2 & 10 \\
\hline
\end{tabular}

El cuadro $\mathrm{N}^{0} 8$, se puede apreciar que, en relación al nivel de empleo del merchandising de la publicidad en la difusión de recursos turísticos, existe una inclinación en un $40 \%$ en empleo abundante y $30 \%$ en empleo frecuente, en relación a un $10 \%$ que emplea mínimamente.

Tabla $N^{\circ}$ 9. Cual es el nivel de empleo de la participación en eventos en la publicidad y la difusión de recursos turísticos.

\begin{tabular}{lcc}
\hline \multicolumn{1}{c}{ Condición } & & $\%$ \\
\hline Emplea abundantemente & 1 & 5 \\
Emplea frecuentemente & 3 & 15 \\
Emplea parcialmente & 10 & 50 \\
Emplea minimamente & 6 & 30 \\
\hline
\end{tabular}

El cuadro $N^{0} 1$, se puede apreciar que, en relación al nivel de empleo de la participación en eventos de la publicidad en la difusión de recursos turísticos, existe una inclinación en un $50 \%$ en empleo parcialmente y $30 \%$ en empleo mínimamente, en relación a un $5 \%$ que emplea abundantemente este medio.

Tabla $N^{\circ}$ 10. Nivel de empleo de las relaciones públicas en la publicidad y la difusión de recursos turísticos.

\begin{tabular}{lcc}
\hline \multicolumn{1}{c}{ Condición } & $\%$ \\
\hline Emplea abundantemente & & \\
Emplea frecuentemente & 5 & 25 \\
Emplea parcialmente & 12 & 60 \\
Emplea mínimamente & 3 & 15 \\
\hline
\end{tabular}

El cuadro $N^{0} 10$, se puede apreciar que, en relación al nivel de empleo de las relaciones públicas de la publicidad en la difusión de recursos turísticos, existe una inclinación en un $60 \%$ en empleo parcialmente y $25 \%$ en empleo frecuente, en relación a un $15 \%$ que emplea este medio mínimamente. 


\section{DISCUSIÓN}

En Perú se ha desarrollado desde hace varios años, dándose fuerza en su implementación a partir del 2000. La agencia de publicidad Grey ha sido la pionera en desarrollar divisiones de BTL, tanto así que muchos clientes calificados como grandes con marcas líderes en el mercado destinan el 50\% a $60 \%$ de sus presupuestos a BTL, conservando dentro de su plan estratégico el ATL Dentro de ellas tenemos el caso de la Corporación Backus quien destina una fuerte inversión para actividades de BTL.

Supongamos que ponemos un aviso en el diario, en la sección de citas. Esto sería usar un medio ATL para lograr la atención de una persona. El mensaje que doy en dicho aviso debe ser de interés de las personas con quienes podría tener más afinidad, quienes le podrá gustar lo que nosotros podemos ofrecer. Si queremos tener éxito con nuestro aviso entendemos que no todos serán de nuestro gusto, ni tampoco nosotros seremos de su gusto, por lo que diseñamos un mensaje que apele al gusto de algunos.

Otra cosa importante del mensaje, es que no tratamos de hacer una "venta" al primer contacto, es decir, no le pedimos a todos quienes leen nuestro aviso que se casen con nosotros, ya que sólo una persona loca haría tal cosa, no? Sino que dejamos los suficientes datos como para que las personas interesadas nos pueda contactar y así poder concretarse una cita. Obviamente también contamos una historia para vender nuestra imagen.

Hasta esta instancia la "publicidad" ATL ha hecho lo que puede hacer, nos ayudó a transmitir un mensaje, que según cómo se haya diseñado o redactado generó que ciertas personas se interesaban por la imagen que se estaban formando de nosotros, sienten que tienen una idea de cómo somos y qué le podemos ofrecer como posible pareja.

Esto trasladando al entorno turístico no da la posibilidad innegable de su utilización como un medio alternativo de publicidad, lo cual se puede hacer uso sin mayor dificultad, considerando la posibilidad financiera de la organización, en estos últimos tiempos existe una competencia por cada uno de los encargados de operar y explotar racionalmente un espacio turístico

Below the line, traducido al español significa debajo de la línea, más conocido por su acrónimo BTL, es una técnica de Marketing consistente en el empleo de formas de comunicación no masivas dirigidas a segmentos específicos, desarrollada para el impulso o promoción de bienes o servicios mediante acciones debe ser caracterizada por el empleo de altas dosis de creatividad y sentido de oportunidad, creándose de ésta manera novedosos canales para comunicar mensajes.

Dentro de sus ventajas se podría decir que en muchos de sus casos su implementación es de costo bajo, haciéndolo accesible a anunciantes que disponen de pocos recursos económicos para solventar una campaña ATL.

\section{REFERENCIAS BILIOGRÁFICAS}

1. Acerenza, Miguel. Promoción turística. Trilla. 1ra edición. México, 1995.

2. Boullon, Roberto C. Los municipios turísticos, Trillas, 1ra edición. México, 1995.

3. Begazo, José D. Mega tendencias del turismo en el tercer milenio, San Marcos, Perú, 2002

4. Borrero Briceño. Fundamentos de marketing, San Marcos, 1ra edición. Perú, 1998.

5. Foster, Dennis L. Ventas y mercadotecnia para el profesional de turismo, Mc. Grow Hill, 2da edición. México. 1994.

6. Hernandez Dial E. Proyectos turísticos, formulación y evaluación, Trillas, México HAUYÓN DALL'ORTO, José. 2000. Turismo en el nuevo milenio, USMP, 1ra edición. Perú. 1983.

7. Lundberg, D. E. Organización y administración de turismo, Centrum, New York SOSA DE LA CRUZ, 2000. Clifor. Los servicios turísticos, cualidades y medición, San Marcos, Perú, 1994. 